\title{
LA RICERCA-AZIONE E I SUOI CONTRIBUTI ALLA SCIENZA METODOLOGICA: ASPETTI GENERALI
}

\section{ARTICOLO ORIGINALE}

DENDASCK, Carla Viana ${ }^{1}$

DENDASCK, Carla Viana. La ricerca-azione e i suoi contributi alla scienza metodologica: aspetti generali. Revista Científica Multidisciplinar Núcleo do Conhecimento. Anno. 06, Ed. 11, Vol. 11, pp. 118-135. Novembre 2021. ISSN: 24480959, Link di accesso: https://www.nucleodoconhecimento.com.br/formazioneit/scienza-metodologica, DOI: 10.32749/nucleodoconhecimento.com.br/formazioneit/scienza-metodologica

\section{RIEPILOGO}

A causa dell'aumento della popolarità e della possibilità di utilizzare la ricerca-azione, lo strumento ha iniziato a essere utilizzato in modo significativo, ma con un concetto non ancora consolidato, il termine è stato applicato in modo astratto, senza interpretazioni profonde e dettagliate dell'uso in vari contesti. Si intende chiarire in tutto questo articolo il significato del termine, e come questo tipo di strumento possa essere applicato nel migliore dei modi, agendo nell'ambito del rigore scientifico-metodologico. Il problema della ricerca è: quali sono le possibilità di utilizzare la ricerca-azione, le sue fasi e le cure fondamentali? La rilevanza dello strumento sta nel fatto che consente lo svolgimento di una ricerca in modo sistematizzato, continuo ed empiricamente basato. Pertanto, verrà discusso il ruolo della teoria nella ricerca d'azione e verranno evidenziate le caratteristiche inerenti alle sue fasi fondamentali. Dovrebbero essere considerate alcune domande comuni relative al metodo, come la partecipazione del ricercatore, la funzione sociale di riflessione, la necessità di gestire le conoscenze acquisite e l'etica nella ricerca. Infine, vengono presentati alcuni "modelli" di ricerca-

\footnotetext{
${ }^{1}$ Teologo, Dottorato di Ricerca in Psicoanalisi Clinica. Lavora da 15 anni con metodologia scientifica (metodo di ricerca) nella guida alla produzione scientifica di studenti di master e dottorato. Specialista in ricerche di mercato e ricerche incentrate sulla salute. Dottorando in Comunicazione e Semiotica (PUC SP).

RC: 102351

Link di accesso: https://www.nucleodoconhecimento.com.br/formazione-it/scienzametodologica
} 
azione che possono contribuire ai ricercatori nella scelta metodologica e nell'organizzazione.

Parole chiave: Ricerca di azioni, Metodi di ricerca, Strumenti di ricerca.

\section{INTRODUZIONE}

La ricerca d'azione è un tipo di ricerca che richiede, da parte del partecipante, una postura impegnata (TRIPP, 2005). È una strategia, in un certo senso, opposta alla ricerca tradizionale, che è considerata "indipendente", "non reattiva" e "oggettiva" (ENGEL, 2000). Quindi, come indica il termine stesso, la ricerca-azione mira a unire la ricerca con l'azione, cioè la pratica. Pertanto, la conoscenza e la comprensione sono intese come parte della pratica, ed è quindi un modo per impiegare la ricerca in situazioni in cui il ricercatore ha anche un'inclinazione pratica e desidera migliorare la comprensione di una teoria (KOERICH et al, 2009). Gli argomenti contrari alla ricercaazione sono dovuti in particolare al fatto che il ricercatore è coinvolto nel campo studiato, che sarebbe in linea con i presupposti di etica nella ricerca scientifica che raccomanda l'esenzione del ricercatore come parte del processo di qualità scientifica, alludendo al fatto che questa esenzione sarebbe responsabile di portare chiarezza negli argomenti del ricercatore che sarebbe assente dalla tendenza nella produzione di risultati (TRIPP, 2005; KOERICH et al, 2009).

Questo tipo di ricerca è nato dalla necessità di superare un divario esistente tra gli assi della teoria e della pratica. Una delle sue caratteristiche predominanti è che si tratta di una ricerca da cui cerca di intervenire nella pratica in modo innovativo durante lo sviluppo della ricerca. Non è solo una raccomandazione che appare nella fase finale (ENGEL, 2000). Questo aspetto è stato esplorato soprattutto da programmi che mirano a promuovere il pragmatismo come prodotto finale, come nei corsi di laurea in ingegneria, business o anche nei programmi di master e dottorato professionali.

Uno dei primi a introdurre la ricerca-azione nel contesto accademico è stato lo psicologo tedesco Kurt Lewin. Tuttavia, già nel 1960, la sociologia si è rapidamente appropriata del concetto (TRIPP, 2005). Si presumeva che il ricercatore dovesse RC: 102351

Link di accesso: https://www.nucleodoconhecimento.com.br/formazione-it/scienzametodologica 
abbandonare questa postura più passiva e isolata, assumendo così le conseguenze causate dai risultati della sua ricerca, e che tali risultati dovessero essere messi in pratica, soddisfacendo così una richiesta che cercava un maggiore contributo dal contesto accademico al mondo pratico (FRANCO, 2005).

Questa ricerca nasce quindi nel cambiamento della postura teorica, in una postura pratica che può interferire nel corso degli eventi. In questo contesto, oltre alla sua applicabilità nelle scienze sociali e nella psicologia stessa, oggi, le sue possibilità di utilizzo sono ampie, il che giustifica la rilevanza di questo studio. Una delle sue nuove possibilità è l'uso nel settore dell'istruzione, sviluppato come risposta alle richieste tangenziali all'implementazione della teoria educativa nella pratica dell'insegnamento, cioè nelle aule (ENGEL, 2000). Oggi, uno dei risultati della ricerca-azione nell'universo dell'educazione, può essere trovato attraverso i numerosi materiali che lavorano con metodologie attive, anche potenziando, in modo che il concetto di metodologia attiva sarà incorporato da alcuni ricercatori come disciplina e metodologia indipendente.

Come accennato in precedenza, la teoria e la pratica non erano percepite come parti intrinseche della vita professionale. Di fronte a tali cambiamenti, la ricerca-azione ha iniziato a essere implementata nei contesti più diversi (TRIPP, 2005). L'obiettivo è quello di aiutare ricercatori e professionisti a risolvere i problemi che riguardano la loro pratica lavorativa quotidiana, facendosi coinvolgere molto di più nella ricerca (MIRANDA; RESENDE, 2006). Con questo, nel campo dell'educazione, i valori, le percezioni e le credenze nell'esercizio dell'insegnamento-apprendimento sono stati valutati empiricamente (ENGEL, 2000). Resta inteso che questo tipo di ricerca è stato visto con occhi buoni dagli accademici più diversi. È una ricerca interessante perché porta il ricercatore a un risultato specifico e immediato in relazione a un determinato problema di ricerca (GRITTEM; MEIER; ZAGONEL, 2008).

Si dice che la ricerca-azione si è dimostrata uno strumento efficace per lo sviluppo professionale di diversi ricercatori. Si sottolinea che questo processo avviene "dall'interno verso l'esterno", poiché parte delle preoccupazioni e degli interessi delle persone coinvolte nella pratica della ricerca (TRIPP, 2005). I soggetti sono coinvolti in 
questioni che favoriscono il proprio sviluppo professionale e anche lo sviluppo personale. L'approccio opposto alla ricerca-azione è proprio la ricerca tradizionale, perché la ricerca si svolge da "fuori a dentro" (ENGEL, 2000). In questo processo, canonico, quindi, il ricercatore alimenta il suo studio con le percezioni portate da un'esperienza esterna, e questa esperienza viene raccolta attraverso alcuni strumenti di ricerca (come questionari e interviste) (FRANCO, 2005). Tuttavia, non vi è alcun obiettivo per discutere un approccio ideale, in quanto si tratta di proposte uniche e diverse. Questi sono due modi di guardare alla natura della ricerca scientifica.

Si presume che esistano verità scientifiche nel mondo al di fuori della ricerca, e spetta allo scienziato scoprire tali verità, compresa la capacità di intervenire per testare tali verità. Tuttavia, nella ricerca scientifica, è deducibile che ci sono modi di guardare alla natura di una ricerca, in modo che non ci siano verità scientifiche assolute, perché tutta la conoscenza è provvisoria e cambia a seconda del contesto storico in cui si vive (MIRANDA; RESENDE, 2006).

I fenomeni sono osservati e interpretati secondo tali particolarità storiche e culturali. Si dice anche che i modelli di ricerca stessi sono soggetti a cambiamenti, perché la scienza si evolve ogni giorno. Non esiste una metodologia scientifica universale e storica, ma modi distinti di guardare il mondo sotto pregiudizi scientifici (GRITTEM; MEIER; ZAGONEL, 2008). La ricerca-azione, in questo senso, si avvicina a una visione più pratica dei fenomeni e delle situazioni quotidiane. Pertanto, la conoscenza scientifica è considerata provvisoria e dipendente da un contesto.

Considerando la sfera dell'educazione, si comprende che gli insegnanti, invece di essere semplici consumatori di una ricerca condotta da altri, dovrebbero trasformare la propria pratica di insegnamento attraverso i risultati di questo studio (ENGEL, 2000). Così, la ricerca-azione è concepita come lo strumento ideale per l'esecuzione di una ricerca che vuole unire la teoria con la pratica. In questo senso, l'obiettivo generale di questa ricerca è, attraverso uno studio esplorativo e descrittivo, riflettere sulle possibilità di applicazione della ricerca-azione in qualsiasi ambiente di interazione sociale. II problema della ricerca è: quali sono le possibilità di utilizzare la ricerca- 
azione e i suoi passaggi e cure fondamentali? Sulla base del problema, mira a verificare come un ambiente in cui si verifica l'interazione sociale e che ha un problema reale e urgente possa beneficiare di questo tipo di studio, un ambiente che coinvolge persone, compiti e procedure che hanno bisogno di aiuto.

\section{LA RICERCA-AZIONE E LA SUA INFLUENZA SULLA PRATICA SOCIALE}

La letteratura descrive la traiettoria della ricerca d'azione dal 1940. Da un punto di vista generale, questo può essere collocato in due periodi significativi: il primo è collegato a una corrente americana, segnata dal l'emergere del termine coniato da Kurt Lewis nel periodo precedente la seconda guerra mondiale (MIRANDA; RESENDE, 2006). Questa fase dura fino al 1960. II secondo momento, a sua volta, è segnato da una corrente europea, corrente che copre il periodo dal 1960 ai giorni nostri (BARBIER, 2002; MORIN, 2004). I due filoni presentano una panoramica di questo tipo di ricerca, concentrandosi sull'approccio qualitativo impiegato dalla ricerca sulle scienze sociali. I concetti, le giustificazioni e le spiegazioni metodologiche costruite attraverso collegamenti teorico-metodologico hanno delimitato la ricerca sinora svolta (BARBIER, 2002; MORIN, 2004).

Da ciò nasce la nozione di intervento, che può variare da una posizione più legata agli studi sperimentali a progetti di azione sociale, il cui scopo è la risoluzione dei più diversi problemi sociali (THIOLLENT, 1984). A causa dei vari concetti e autori che hanno iniziato a discutere di ricerca-azione, diverse letture e interpretazioni sono diventate costanti, propendendo ora per una prospettiva più esplicativa (sperimentale), o per una più completa (fenomenologica o dialettica) (TRIPP, 2005). La ricerca-azione emerge come una critica, inizialmente, al positivismo, anche se si comprende che non tutti si sono allontanati da tale comprensione. In linea di principio, sono anche visti come approcci di natura globale, perché riconoscono la realtà sociale come qualcosa che non esiste o può essere riconosciuto come indipendente e autonomo (FRANCO, 2005). È, quindi, una realtà soggettiva e, come tale, è costruita e sostenuta attraverso atti individuali.

RC: 102351

Link di accesso: https://www.nucleodoconhecimento.com.br/formazione-it/scienzametodologica 
Sono questi atti individuali che attribuiscono significati e significati a questa realtà nel processo di costruzione (CARR; KEMMIS, 1988). Tra gli altri aspetti, si può anche menzionare che esiste un parallelo tra queste nuove riletture della ricerca-azione. Gli approcci francese e canadese, proposti, rispettivamente, da René Barbier (2002) e André Morin (2004), si connettono. Prendendo come riferimento questi autori, così come alcune delle loro opere, non si intende generalizzare il loro pensiero e la forma di produzione di questo tipo di studio, ma piuttosto elencare alcuni dei suoi elementi generali, perché questi ci permettono di comprendere, in modo profondo, lo svolgimento della ricerca d'azione nei contesti più diversi (TRIPP, 2005). II campo dell'educazione, come detto, è una delle possibilità di spazi in cui la ricerca-azione può agire in modo significativo. Inoltre, la ricerca-azione è attribuita a un significato: è classificata come una rivoluzione epistemologica (BARBIER, 2002).

Si chiama così, perché fino ad allora la proposta non era stata esplorata in modo significativo nel campo delle scienze umane. Pertanto, si è capito che la ricerca-azione era una sorta di "arte del rigore clinico" (BARBIER, 2002). Pertanto, può essere sviluppato con una collettività. L'obiettivo, quindi, è quello di analizzare una porzione di questa società in modo meno distante, meno oggettivo, come avviene nella ricerca tradizionale, in cui si difende la neutralità e la presa di distanza dal ricercatore (TRIPP, 2005). Tuttavia, affinché questo tipo di studio diventi fattibile, implica un cambiamento della postura del soggetto, sia esso un individuo o un gruppo sociale, rispetto alla loro realtà (KOERICH et al, 2009). È quindi necessario fornire percezioni e opinioni reali su un determinato fenomeno vissuto quotidianamente. II ricercatore, quindi, cattura questo fenomeno e lo registra, unendo queste percezioni, valori e credenze del soggetto / gruppo analizzato con le loro concezioni teoriche.

L'esercizio della ricerca-azione implica che il ricercatore abbia una visione sistemica aperta per registrare i fenomeni osservati. Pertanto, al momento della registrazione, deve combinare determinati processi, come l'organizzazione, l'informazione, le percezioni, i valori, le credenze, con le fonti da cui parte per costruire il suo studio secondo il rigore scientifico richiesto (MIRANDA; RESENDE, 2006). La vicinanza del ricercatore del fenomeno indagato è uno degli aspetti che allontana la ricerca d'azione RC: 102351

Link di accesso: https://www.nucleodoconhecimento.com.br/formazione-it/scienza$\underline{\text { metodologica }}$ 
da quelle più canoniche, teoriche, solo. Ciò è dovuto alle stesse ipotesi che sono alla base di questo strumento sin dal momento della sua creazione (ENGEL, 2000). Gli autori pionieri nel campo, nei loro studi, criticano l'azione del positivismo nel campo delle scienze sociali, poiché questa tendenza ha introdotto limiti all'attività di ricerca, e questo ha iniziato a basarsi su alcuni pilastri, come l'obiettività, la razionalità e la verità (KOERICH et al, 2009).

La ricerca-azione è legata all'idea di cambiamento costante e, quindi, si basa sul presupposto che anche gli attori che costituiscono la realtà sono in un costante processo di trasformazione (TRIPP, 2005). In considerazione di questa mentalità, è nata la necessità di pensare al concetto di ricerca insieme all'azione, cioè alla pratica, e, con questo, abbiamo iniziato a riflettere sulle strategie di risoluzione dei problemi sociali da un altro approccio (FRANCO, 2005). In questo contesto, gli studi che scelgono questo approccio di ricerca conciliano i campi della pratica, dell'azione sociale, con quelli teorici, epistemologici. La pratica, cioè l'azione, riguarda tutto ciò che è costituito e mantenuto in un determinato contesto e, con questo, copre anche l'azione e l'esperienza quotidiana di un soggetto (GRITTEM; MEIER; ZAGONEL, 2008). La ricerca-azione è più di un approccio metodologico. Questa è una posizione di fronte a questioni epistemologiche essenziali, come la relazione tra soggetto e oggetto, teoria e pratica.

\section{CARATTERISTICHE DI UNA RICERCA-AZIONE}

L'action research comprende un processo empirico, che consente l'identificazione del problema all'interno di un contesto sociale e/o istituzionale, la raccolta di dati relativi al problema in questione e l'analisi dei significati e dei significati attribuiti ai dati relativi ai partecipanti (TRIPP, 2005). Oltre a identificare la necessità di cambiamento e a rilevare le possibili soluzioni necessarie, la ricerca-azione interviene anche nell'ambiente pratico, in quanto provoca e guida la trasformazione (ENGEL, 2000). È uno strumento metodologico in grado di coniugare teoria e pratica attraverso un'azione che mira alla trasformazione di un determinato contesto, cioè di una certa realtà (MIRANDA; RESENDE, 2006). La ricerca-azione, quindi, consente di associare, al 
processo di ricerca, la possibilità di apprendimento, poiché richiede il coinvolgimento creativo e consapevole sia del ricercatore che degli altri membri della ricerca (ROLIM et al, 2004).

In questo contesto, l'importanza della ricerca-azione emerge come strumento multidisciplinare, poiché, allo stesso tempo, opera in diversi campi, come nel settore sanitario, nel campo dell'istruzione, negli ambienti caratterizzati da innovazione e cambiamento, tra gli altri (TRIPP, 2005). Diversi gruppi possono essere contemplati, come professionisti, manager, studenti e la popolazione generale, sia nelle comunità che nelle istituzioni (ROLIM et al, 2004). Ci sono alcune caratteristiche che hanno delimitato la ricerca-azione, come la concettualizzazione dei problemi che verranno lavorati, la pianificazione, l'esecuzione e la valutazione delle azioni per risolverli. C'è, quindi, la ripetizione di questo ciclo di attività (ANDRÉ, 2000). Oltre al suo famigerato contributo sociale, il lavoro di Lewin sulla ricerca-azione è stato considerato innovativo, in quanto promuove, allo stesso tempo, il carattere partecipativo e democratico, poiché la ricerca si sviluppa dalla partecipazione dei soggetti studiati (TRIPP, 2005).

La ricerca-azione adotterà i seguenti processi nella sua costruzione:

Tabella 1 - Fasi della ricerca-azione 


\section{ETAPAS DA PESQUISA AÇÃO}

\section{PESQUISA}

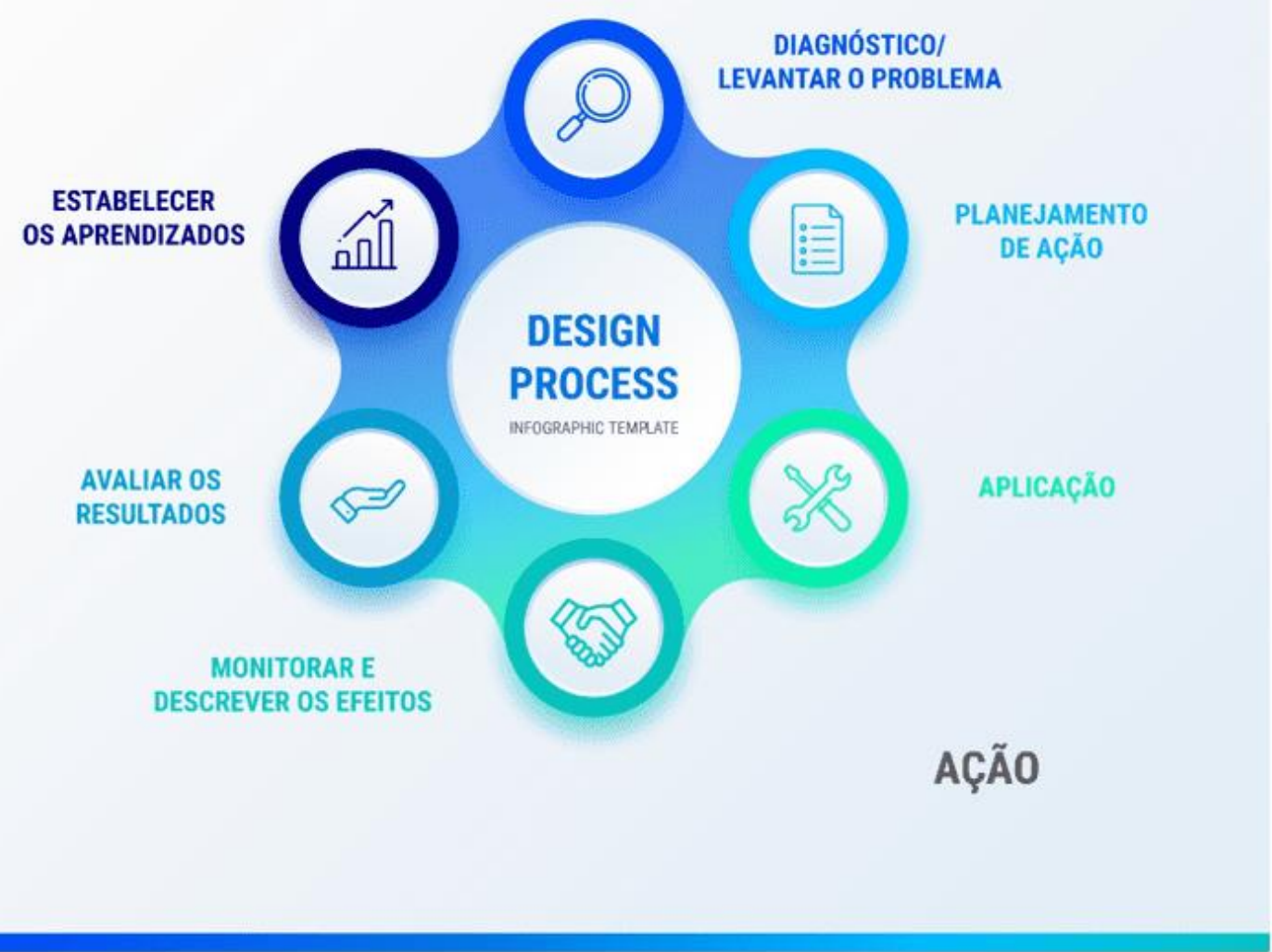

Fonte: Autore.

Pertanto, esiste uno studio volto a risolvere problemi che riguardano un determinato ambiente, concentrandosi, quindi, sulle specificità di questo contesto, dove questi problemi si manifestano (PEREIRA, 2001; LEWIN, 1946). Un'altra caratteristica di questo tipo di ricerca è la preoccupazione per la validità scientifica dei risultati ottenuti dai collaboratori. In questo processo, vengono prese in considerazione le diagnosi, in quanto sottolineano la situazione prima e dopo l'uso di determinate azioni, oltre a consentire la registrazione dettagliata di tutti gli eventi (LEWIN, 1946). La comprensione dello strumento di ricerca qui discusso è legata a due concetti

$\mathrm{RC}: 102351$

Link di accesso: https://www.nucleodoconhecimento.com.br/formazione-it/scienzametodologica 
fondamentali, che sono l'atto di indagine e l'atto sostanziale. L'atto di indagine è un'azione in grado di stimolare e promuovere un'indagine (TRIPP, 2005). L'atto nominale, a sua volta, è un'azione in grado di promuovere un cambiamento desiderato in un determinato contesto osservato e studiato.

Si tratta, quindi, di un processo che unisce la ricerca all'azione (KOERICH et al, 2009). In questo senso, nell'indagine dell'azione, gli atti sono necessariamente caratterizzati come sostanziali. Pertanto, a causa di questa qualifica, l'atto di indagare presuppone un obbligo, che è la necessità di beneficiare le persone che non appartengono alla comunità scientifica, ma possono beneficiare dei risultati di uno studio scientifico (LEWIN, 1946). Così, la scienza, dai suoi ricercatori, assume una nuova missione: far sì che la conoscenza prodotta nelle università, nazionali o straniere, raggiunga la società secolare, in modo che i soggetti che compongono i gruppi sociali più diversi siano emancipati (FRANCO, 2005). La ricerca-azione ideata da Lewin è stata utilizzata in modi diversi e con scopi diversi, dando vita a un ampio mosaico di approcci teoricometodologici.

Ci sono almeno tre diversi concetti applicati alla ricerca-azione in Brasile. Sono classificati in base ad alcuni criteri. II primo tipo è la ricerca collaborativa (la trasformazione è richiesta dal gruppo di riferimento al team di ricerca) (KOERICH et al, 2009). II ruolo del ricercatore, in questo processo, è quello di essere parte di un cambiamento, reso scientifico. Questo è innescato dai soggetti che fanno parte del gruppo (TRIPP, 2005). C'è anche una ricerca critica sull'azione. La necessità di trasformazione è percepita dagli studi iniziali del ricercatore con il gruppo (FRANCO, 2005). Quando il cambiamento deriva da un processo che valorizza la costruzione cognitiva dell'esperienza, supportata dalla riflessione critica collettiva, al fine di emancipare i soggetti ed eliminare condizioni che il collettivo considera oppressive, la ricerca assume un carattere essenzialmente critico (ENGEL, 2000). C'è anche la ricerca strategica d'azione.

Se negli altri approcci la trasformazione è precedentemente pianificata, senza l'azione attiva dei soggetti, lo stesso non accade qui. Se c'è un solo ricercatore responsabile 
della valutazione e del monitoraggio dei risultati dell'applicazione dello studio, la ricerca action-critical perde il suo bias critico (FRANCO, 2005). Affinché la ricerca critica sull'azione sia caratterizzata in questo modo, è necessario un calo nella prassi del gruppo sociale analizzato, il cui obiettivo è quello di estrarre le prospettive latenti che supportano le pratiche, cioè i cambiamenti sono negoziati e amministrati nel collettivo (KOERICH et al, 2009). La ricerca collaborativa, in questo contesto, assume anche un carattere critico (THIOLLENT, 1984). La criticità che scaturisce da questo tipo di studio richiede un processo di riflessione collettiva sulle strategie operative da adottare. Questo processo considera la voce del soggetto, così come le sue prospettive. L'interesse non solo per il disco e per un'interpretazione successiva.

È una parte inseparabile di questa metodologia investigativa, in modo che non possa essere stabilita attraverso passaggi lineari mirati al metodo stesso, ma sia organizzata da situazioni e fatti che emergono nel processo e diventano essenziali (ENGEL, 2000). Emerge l'enfasi sul carattere formativo di questo tipo di ricerca, in modo che il soggetto diventi consapevole delle trasformazioni che influenzano sia il ricercatore che il gruppo e il processo (TRIPP, 2005). A causa di questo obiettivo, la ricerca-azione assume un carattere emancipatorio, in modo che i soggetti coinvolti nella ricerca possano liberarsi da miti e pregiudizi che impediscono l'arrivo dei cambiamenti desiderati (BARBIER, 2002). Questo tipo di ricerca, nel corso degli anni, come detto, è stato influenzato dalle correnti positiviste, poiché la dialettica della realtà sociale è stata incorporata nella vita quotidiana, così come i punti che sostengono e sostengono la diffusione di una razionalità critica lineare.

In questo scenario, lo status epistemologico che sostiene la ricerca-azione ha iniziato a preoccuparsi dei processi alla base della trasformazione sociale, impegnandosi negli aspetti etici e politici legati all'emancipazione dei soggetti, concentrandosi anche sulle condizioni che supportano il processo di emancipazione (MIRANDA; RESENDE, 2006). La ricerca-azione ha iniziato ad ammettere alcuni approcci interpretativi all'analisi e ha ricevuto una struttura in grado di favorire la partecipazione critica delle persone coinvolte, che ha indotto il processo di ricerca ad ammettere le ricostruzioni, nonché le risignificazioni di significati e percorsi durante l'esecuzione delle fasi (TRIP RC: 102351

Link di accesso: https://www.nucleodoconhecimento.com.br/formazione-it/scienza$\underline{\text { metodologica }}$ 
$P$, 2005). Ha assunto una forma caratterizzata come pedagogica e politica (THIOLLENT, 1984). Nel contesto della ricerca qualitativa, ci sono tre dimensioni che dovrebbero essere considerate dalla ricerca d'azione. II primo di questi è ontologico. Riguarda la natura dell'oggetto da conoscere e indagare dal ricercatore.

La seconda dimensione è epistemologica e riguarda il soggetto che cerca di conoscere. La terza dimensione, infine, è la dimensione metodologica, il cui obiettivo è conoscere i processi da cui la conoscenza è stata costruita dal ricercatore coinvolto in un determinato gruppo (FRANCO, 2005). Per quanto riguarda la dimensione ontologica, si può dire che essa è legata a una conoscenza guida, e questo dovrebbe consentire ai soggetti di produrre conoscenza per una migliore comprensione degli elementi che condizionano una certa prassi sociale (KOERICH et al, 2009). Gli scopi di questa ricerca sono desiderati dal collettivo stesso, che richiede, al ricercatore, la necessità di produrre conoscenze che possano consentire la ristrutturazione di determinati processi formativi (FRANCO, 2005). Per quanto riguarda la dimensione del carattere epistemologico, si ricorda che, per il suo esercizio, il ricercatore si immerge nell'intersoggettività della dialettica del collettivo.

Pertanto, il ricercatore deve assumere una postura differenziata quando si tratta e interpreta la conoscenza, poiché, allo stesso tempo, cerca di conoscere e intervenire nella realtà di quel gruppo che viene indagato (ENGEL, 2000). L'unione tra ricerca e azione, quindi, fa sì che il ricercatore debba essenzialmente entrare nell'universo ricercato, ma questa necessità non annulla in alcun modo la possibilità di adottare una postura neutrale quando si tratta dei dati, perché questo è un presupposto della scienza stessa (KOERICH et al, 2009). La neutralità è un modo di controllare le circostanze che permeano una ricerca, il che implica attirare l'attenzione sui presupposti epistemologici fondamentali, come la prioritizzazione della dialettica della realtà sociale e la storicità dei fenomeni, la prassi, le contraddizioni, le relazioni con la totalità e l'analisi dell'azione dei soggetti sulla base di alcune circostanze $(\mathrm{KOERICH}$ et al, 2009). 
La prassi, in questo contesto, dovrebbe essere concepita come un modo fondamentale di costruire la conoscenza, perché, attraverso di essa, teoria e pratica sono articolate (FRANCO, 2005). Per quanto riguarda la conoscenza da analizzare, questa non si limita alla mera descrizione, perché spetta al ricercatore spiegare tali trasformazioni da ciò che osserva, cioè attraverso un movimento che integra la dialettica del pensiero e dell'azione (TRIPP, 2005). Di conseguenza, la conoscenza derivata da questa relazione è in grado di trasformare i soggetti e le circostanze relative al mezzo di cui fa parte. La dimensione metodologica, a sua volta, richiede alcune procedure che articolano l'ontologia con l'epistemologia della ricerca d'azione, il che implica stabilire nel gruppo osservato una dinamica che integri i principi e le pratiche dialogiche, partecipative e trasformative (TRIPP, 2005). In questa dimensione, alcuni elementi devono essere presi in considerazione.

Tra questi, si può menzionare la prassi. È il punto di partenza e di arrivo quando si tratta della costruzione e/o della risignificazione della conoscenza (FRANCO, 2005). Si basa sulla nozione che la conoscenza può essere costruita solo attraverso molteplici articolazioni con intersoggettività. In considerazione di questa caratteristica, la ricercaazione, per essere ben articolata, deve essere condotta nell'ambiente naturale della realtà da ricercare, in modo che i risultati raccolti siano veri, sicuri e di qualità (ENGEL, 2000). In questo processo, c'è una certa flessibilità, poiché l'obiettivo è catturare una realtà nel processo di cambiamento. Pertanto, la metodologia dovrebbe essere in grado di consentire al ricercatore di apportare determinati aggiustamenti e il ricercatore dovrebbe camminare secondo la sintesi provvisoria. Tali sintesi sono stabilite nel gruppo stesso (GRITTEM; MEIER; ZAGONEL, 2008). Sono questi elementi che sottolineano come un certo contesto sia stato influenzato.

II metodo dovrebbe comportare l'esercizio continuo di spirali cicliche. Queste spirali tengono conto di alcuni passaggi fondamentali, come la pianificazione, l'azione, la riflessione, l'atto di ricerca, la risignificazione e la ripianificazione, quest'ultima quando necessario (KOERICH et al, 2009). Per quanto riguarda la ricerca-azione, si evidenzia anche che, mentre un processo investigativo, l'educazione e l'azione avvengono contemporaneamente. In questo senso, le ragioni epistemologiche e metodologiche RC: 102351

Link di accesso: https://www.nucleodoconhecimento.com.br/formazione-it/scienzametodologica 
che permeano l'attività investigativa fanno sì che la conoscenza sia prodotta attivamente, perché tutti coloro che sono coinvolti nel processo devono collaborare (ENGEL, 2000). L'indagine, in questo contesto, consente di comprendere i cambiamenti nella realtà attraverso l'azione (TRIPP, 2005). II processo richiede una postura specifica da parte del ricercatore.

Spesso si presenta con certi tipi di domande, così come con alcune richieste specifiche, come l'inserimento nella cultura che sta analizzando nuovi significati, significati, rappresentazioni, resistenze, aspettative ed esperienze (FRANCO, 2005). La sfida che il ricercatore si trova ad dovere, a sua volta, è quella di creare un nuovo ambiente, in un processo di costruzione familiare. In altre parole, il cambiamento dovrebbe essere percepito da tutti i soggetti coinvolti (KOERICH et al, 2009). II ricercatore è uno dei partecipanti dell'universo che viene indagato, tuttavia, la neutralità della ricerca è un principio che dovrebbe essere considerato. Pertanto, le loro percezioni sul fenomeno analizzato non possono essere ignorate, tuttavia, la sua gestione deve essere fatta nel modo appropriato. Uno degli obiettivi che possono essere raggiunti attraverso questa strategia riguarda il raggiungimento, tra i partecipanti, di una maggiore fiducia (KOERICH et al, 2009).

II ciclo di ricerca-azione inizia con la fase esplorativa. In esso, viene fatta la diagnosi sulla realtà e viene elaborata un'indagine sul contesto, i problemi iniziali e le possibili azioni (TRIPP, 2005). Da questo, ricercatori e partecipanti stabiliscono gli obiettivi principali della ricerca. Questi dovrebbero essere collegati al campo osservato, agli attori e ai tipi di azioni in cui si intende concentrarsi sull'attività investigativa (FRANCO, 2005). II tema della ricerca è definito in sequenza. Questo è delimitato da un problema pratico relativo a un'area di ricerca. Questo viene scelto in base agli impegni assunti tra il team di ricercatori e i soggetti che corrispondono alla situazione in questione (FRANCO, 2005). Tuttavia, il tema può anche essere richiesto dagli attori della situazione. Dovrebbe essere di interesse sia per i ricercatori che per i soggetti indagati in modo che tutti prendano un ruolo attivo e contribuiscono allo sviluppo della ricerca. 
Sceglie anche un quadro teorico specifico con cui lavorare, perché guiderà tutta la ricerca. In terzo luogo, vengono eletti i problemi, cioè il problema della ricerca. È dal problema che il tema acquista robustezza (ENGEL, 2000). II problema dovrebbe riguardare alcune ipotesi di ricerca, come l'analisi e la delimitazione della situazione iniziale; la progettazione della situazione finale, sulla base di criteri di disabilità e fattibilità; l'identificazione di tutti i problemi da risolvere; il recepimento di tali problemi in azioni corrispondenti; infine l'attuazione e la valutazione delle azioni (TRIPP, 2005). In questo senso, è necessario adattare un quadro di riferimenti teorici alla realtà pratica della ricerca. L'obiettivo è che le informazioni siano analizzate e interpretate da una base teorica, tuttavia, devono essere articolate con le esperienze reali, raccolte, quindi, dai collaboratori dello studio (TRIPP, 2005).

Si arriva alle probabilità. Nonostante la falsa idea che non ci siano ipotesi, è necessario riflettere attentamente su questa proposizione, che implica la comprensione delle ipotesi come ipotesi formulate dal ricercatore che possono o non possono essere confermate (KOERICH et al, 2009). Le ipotesi ammettono possibili soluzioni a un problema di ricerca e quindi mirano a condurre una linea di pensiero (KOERICH et al, 2009). II seminario è anche una delle fasi della ricerca-azione, in quanto svolge un ruolo significativo nel processo decisionale in un'indagine, oltre a consentire il coordinamento delle attività. Lo scopo di un seminario è quello di definire il tema e stabilire i problemi con cui la ricerca opererà. L'elaborazione del problema in modo che i problemi siano trattati, così come le ipotesi di ricerca, è uno degli obiettivi. Nascono i gruppi di studio e i gruppi di ricerca che coordinano queste attività.

Le informazioni provengono da diverse fonti e gruppi, che forniscono l'accesso a interpretazioni che favoriscono i processi di definizione delle linee guida d'azione, nonché è possibile valutare le azioni e diffondere i risultati da canali appropriati (KOERICH et al, 2009). La ricerca-azione è anche operazionalizzata dai campi dell'osservazione, del campionamento e della rappresentatività qualitativa. Questo può comprendere una comunità concentrata e/o diffusa, tuttavia, il campionamento e la rappresentatività sono un fattore che può essere discusso. Alcuni studi escludono il campione, altri ne raccomandano l'uso, altri valutano i criteri di rappresentatività RC: 102351

Link di accesso: https://www.nucleodoconhecimento.com.br/formazione-it/scienzametodologica 
qualitativa (FRANCO, 2005). Si entra nella fase di raccolta dei dati. Le principali tecniche utilizzate sono interviste collettive e/o individuali, questionari convenzionali, studio di file, ecc. Dopo la raccolta di informazioni dai gruppi di osservazione, vengono discussi, analizzati e interpretati insieme.

\section{CONSIDERAZIONI FINALI}

Il processo di attività investigativa, l'analisi della realtà, così come la valutazione di strumenti e metodi qualitativi richiedono una certa dialogicità, cioè implica la ricerca di conoscenze riflessive e il rassodamento di un impegno per la realtà concreta. In altre parole, richiede un riconoscimento efficace del soggetto e della realtà in analisi da un movimento dinamico tra le parti coinvolte nell'indagine. Questa possibilità circolare e dinamica sta nella risonanza e nel sostegno alla conquista di un nuovo spazio e/o alla conquista di una nuova conoscenza. Così, la ricerca-azione come strumento metodologico analizza l'azione umana da un movimento comunicativo e partecipativo, che favorisce la condivisione della conoscenza e l'elaborazione di una struttura relazionale di fiducia e impegno verso i soggetti che integrano la realtà da trasformare. Il suo scopo è la risoluzione o il chiarimento dei problemi coinvolti nel gruppo.

Durante il processo, è necessario un monitoraggio continuo delle decisioni, delle azioni e di tutte le attività intenzionali sviluppate dagli attori coinvolti nella situazione. Pertanto, con questo approccio, la ricerca non si limita a una forma di azione, ma solo perché mira ad aumentare la conoscenza dei ricercatori, nonché il loro livello di consapevolezza, sulle persone e / o gruppi coinvolti. La ricerca promuove molto più di una raccolta di dati e/o proposte di interventi pratici. Oltre alle considerazioni qui presentate, si sottolinea che la ricerca d'azione, tra le altre questioni, aggrega discussioni e spiegazioni reali, attuali e coerenti che consentono la generazione di conoscenze descrittive, tuttavia critiche, sulle situazioni vissute in vari ambienti sociali. Promuove, quindi, nuove forme di espressione e riflessione sui significati e sentimenti dei partecipanti attribuiti nell'analisi della situazione problematica in questione. 


\section{RIFERIMENTI}

ANDRÉ, M. E. D. A. Etnografia da prática escolar. Série Prática Pedagógica. Campinas: Papirus; 2000.

BARBIER, R. A pesquisa-ação. Tradução Lucie Didio. Brasília: Plano, 2002.

CARR, W.; KEMMIS, S. Teoria crítica de la enseñanza. Barcelona: Martinez Roca, 1988.

ENGEL, G. I. Pesquisa-ação. Educar em Revista, n. 16, p. 181-191, 2000.

FRANCO, M. A. S. Pedagogia da pesquisa-ação. Educação e pesquisa, v. 31, n. 3, p. 483-502, 2005.

GRITTEM, L.; MEIER, M. J.; ZAGONEL, I. P. S. Pesquisa-ação: uma alternativa metodológica para pesquisa em enfermagem. Texto \& Contexto-Enfermagem, v. 17, n. 4, p. 765-770, 2008.

KOERICH, M. S. et al. Pesquisa-ação: ferramenta metodológica para a pesquisa qualitativa. Revista Eletrônica de Enfermagem, v. 11, n. 3, p. 717-723, 2009.

LEWIN, K. Problemas de dinâmica de grupo. São Paulo: Cultrix, 1946.

MIRANDA, M. G. de.; RESENDE, A. C. A. Sobre a pesquisa-ação na educação e as armadilhas do praticismo. Revista Brasileira de Educação, v. 11, n. 33, p. 511-518, 2006

MORIN, A. Pesquisa-ação integral e sistêmica: uma antropopedagogia renovada. Tradução Michel Thiollent. Rio de Janeiro: DP\&A, 2004.

PEREIRA, E. M. A. Professor como pesquisador: o enfoque da pesquisa-ação na prática docente. In: GERALDI, C. M. G.; FIORENTINI, D.; PEREIRA, E. M. A. (Org). Cartografias do trabalho docente: professor(a) - pesquisador(a). Coleção Leituras no Brasil. Campinas: Mercado das Letras, 2001. p. 153-81.

RC: 102351

Link di accesso: https://www.nucleodoconhecimento.com.br/formazione-it/scienza-

metodologica 
ROLIM, K. M. C. et al. Mulheres em uma aula de hidroginástica: experenciando o interrelacionamento grupal. Revista Brasileira em Promoção da Saúde, v. 17, n. 1, p. 8-13, 2004.

THIOLLENT, M. Notas para o debate sobre pesquisa-ação. In: BRANDÃ O, C. (Org.). Repensando a pesquisa participante. São Paulo: Brasiliense, 1984. p. 82-103.

TRIPP, D. Pesquisa-ação: uma introdução metodológica. Educação e pesquisa, v. 31, n. 3, p. 443-466, 2005.

Inviato: Novembre, 2021.

Approvato: Novembre 2021. 